\title{
Evaluación de cualidades del pensamiento de estudiantes de Matemática-Física al ingreso a la universidad
}

Evaluation of the thinking qualities of Mathematics-Physics students upon admission to the university

\author{
Volumen 18, Número 2 \\ Mayo-Agosto \\ pp. 1-23
}

\section{Luis Eduardo Rodríguez Rodríguez \\ María del Carmen Rodríguez Domínguez}

Revista indizada en REDALYC, $\underline{\text { SCIELO }}$

Revista distribuida en las bases de datos:

LATINDEX, DOAJ, REDIB, IRESIE, CLASE, DIALNET, SHERPA/ROMEO, QUALIS-CAPES, MIAR

Revista registrada en los directorios:

ULRICH'S, REDIE, RINACE, OEI, MAESTROTECA, PREAL, CLACSO 


\title{
Evaluación de cualidades del pensamiento de estudiantes de Matemática-Física al ingreso a la universidad
}

\author{
Evaluation of the thinking qualities of Mathematics-Physics students upon admission to the
} university

\section{Luis Eduardo Rodríguez Rodríguez ${ }^{1}$ María del Carmen Rodríguez Domínguez²}

\begin{abstract}
Resumen: El presente artículo científico es resultado de investigaciones realizadas por los autores en el proyecto de didáctica de la Universidad de Ciego de Ávila, Cuba. La problemática se relaciona con la necesidad de elevar los niveles de aprendizaje de los estudiantes en la carrera profesoral de Matemática-Física a partir del estudio del desarrollo del pensamiento lógico. El objetivo trazado es ofrecer una evaluación del nivel de desarrollo de las cualidades del pensamiento de estudiantes de Matemática-Física que ingresan a la universidad, entendidas estas como las características cualitativas que diferencian el pensamiento de una persona. En la investigación se siguió una metodología mixta a partir del diseño e implementación de técnicas experimentales para evaluar el nivel de desarrollo del pensamiento lógico. La población está compuesta por 37 estudiantes de la carrera de Matemática-Física y se trabajó con una muestra de 12 estudiantes que ingresaron en el primer curso en el 2016. Para el análisis de los resultados se determinó el índice promedio y el índice de la muestra para los indicadores definidos, con el fin de valorar el nivel de desarrollo de las cualidades del pensamiento lógico. Los resultados mostraron que la cualidad de consecutividad del pensamiento se encuentra en un nivel alto de desarrollo, mientras que las demás cualidades, en un nivel medio; esto, de acuerdo con los valores de los índices de la muestra que fue de 2,16 para la consecutividad, y se comportaron entre 1,5 y 2 para el resto de las cualidades.
\end{abstract}

Palabras clave: pensamiento lógico, matemáticas, física, educación superior.

\begin{abstract}
This scientific article is the result of a research carried out by the authors in the didactic project of the University Of Ciego de Ávila Cuba. The issue was related to the need to raise the learning levels of students in the career of Mathematics-Physics from the study of the logical thinking development. The aim is to offer an assessment of the level of thinking development qualities of Mathematics'-Physics' students entering the university, understood as the qualitative characteristics that differentiate the thought of a person. In the research a mixed methodology was followed from the design and implementation of experimental techniques to evaluate the level of development of logical thinking. The population 0.37 students of the Mathematics-Physics career and we worked with a sample of 12 students who entered the first course in 2016. For the analysis of the results, the average index and the index of the sample were determined for the defined indicators, in order to assess the level of development of the qualities of logical thinking. The results showed that the quality of consecutiveness of thought is at a high level of development, while the other qualities are at a medium level; this, according to the values of the indices of the sample that was 2.16 for the consecutiveness, and they behaved between 1.5 and 2 for the rest of the qualities.
\end{abstract}

Key words: logical thinking, mathematics, physics, higher education.

\footnotetext{
${ }_{1}^{1}$ Director del Centro de Estudios Educacionales. Universidad de Ciego de Ávila, Cuba. Doctor en Ciencias Pedagógicas. Dirección electrónica: luisrr@sma.unica.cu

2 Profesora e investigadora del Centro de Estudios Educacionales. Universidad de Ciego de Ávila, Cuba. Doctora en Ciencias Pedagógicas. Dirección electrónica: mariacrd@sma.unica.cu
}

Artículo recibido: 12 de mayo, 2017

Enviado a corrección: 18 de octubre, 2017.

Aprobado: 12 de febrero, 2018. 


\section{Introducción}

El desarrollo intelectual del estudiantado en el proceso de enseñanza-aprendizaje de cualquier disciplina es de relevante importancia, de él depende, en gran medida, el futuro técnico y científico de cualquier país; en particular, en el campo de las ciencias naturales y exactas que constituyen la base del desarrollo científico-técnico de la industrialización y la automatización de la mayoría de las ramas de la producción y los servicios. Por esta razón, debe ser una preocupación constante del profesorado de estas disciplinas, la búsqueda de vías y métodos que tiendan al desarrollo intelectual de los estudiantes y a la formación en ellos de modos de actuación acordes con el quehacer científico que les permita desarrollar su pensamiento a niveles elevados.

Es reconocido que el pensamiento se desarrolla ejecutando la acción de pensar y, aunque no de manera absoluta, pensar es esencialmente solucionar problemas (Labarrere, 1988); por tanto, para desarrollar el pensamiento del cuerpo estudiantil habrá que formar en él acciones que le permitan enfrentar exitosamente la resolución de problemas.

La solución de diversos tipos de problemas permite evaluar el desarrollo intelectual de los estudiantes, particularmente, el desarrollo del pensamiento lógico. En la mayoría de las investigaciones realizadas por distintos autores, se considera al problema como el medio más importante para movilizar el pensamiento del sujeto, como la actividad más inteligente del hombre, y se reconoce su trascendencia para el desarrollo de las diferentes operaciones y cualidades del pensamiento y de la personalidad del estudiante de forma general.

El nivel de desarrollo del pensamiento lógico constituye una premisa importante para que los estudiantes logren éxitos en su formación universitaria. De igual modo, conocer el nivel de desarrollo del pensamiento lógico de los estudiantes al ingresar en la universidad por parte de los profesores contribuye a que se diseñen y ejecuten estrategias de enseñanza-aprendizaje acordes a las particularidades individuales de cada uno.

Investigaciones realizadas, por los autores de este artículo, durante varios años demuestran que las cualidades del pensamiento lógico pueden constituirse en dimensiones e indicadores eficaces para valorar el nivel de desarrollo del pensamiento lógico. A partir de estas, es posible elaborar técnicas experimentales que ofrecen resultados confiables en la evaluación del nivel de desarrollo de este importante proceso cognoscitivo (Rodríguez, 2012).

El objetivo de este artículo es ofrecer a la comunidad científica un proceder general para evaluar el nivel de desarrollo de las cualidades del pensamiento lógico en estudiantes 
que ingresan en la carrera de Matemática-Física de la Universidad de Ciego de Ávila. Se siguió en la investigación una metodología mixta a partir de la elaboración y aplicación de técnicas experimentales para evaluar el nivel de desarrollo del pensamiento lógico de los estudiantes.

\section{Fundamentos teóricos para la evaluación del nivel de desarrollo de las cualidades del pensamiento lógico}

La actividad cognoscitiva de la personalidad incluye los niveles siguientes: sensoperceptual (sensaciones y percepciones), representativo (memoria e imaginación) y racional (pensamiento).

En la literatura especializada autores reconocidos, como Rubinstein (1977); Leontiev (1981); Labarrere (1988); Rodríguez, (2012); Flores (2016); Blanco, (2017); Pérez y Merino (2008), Travieso y Hernández (2017), coinciden en caracterizar al pensamiento como el proceso psíquico que es reflejo mediato y generalizado de la realidad objetiva en el cerebro humano. En esta dimensión, el pensamiento es la forma superior de la cognición, dirigido a la búsqueda de lo esencialmente nuevo en los objetos y fenómenos. Mediante el pensamiento es posible el conocimiento de las propiedades, nexos y relaciones esenciales de la realidad y su transformación. Mientras que se asume por pensamiento lógico al pensamiento ordenado, consecuente, que sigue de manera lineal y exacta las acciones planificadas y que no salta aleatoriamente de una idea a otra en la consecución de un fin determinado previamente (Rodríguez, 2012).

De manera sintética, se pueden agrupar los sustentos teóricos acerca de las particularidades del pensamiento lógico, asumidos en la propuesta de dimensiones e indicadores para evaluar el nivel de desarrollo de las cualidades del pensamiento lógico de los estudiantes que se ofrece en el presente artículo, de la siguiente forma:

- Que el pensamiento es un reflejo mediato y generalizado de la realidad: las relaciones reales existentes entre los objetos, hechos y fenómenos de la realidad se reflejan en el pensamiento en forma de conceptos, de juicios, de razonamientos, en la formulación de leyes generales. Se ofrece la definición de las características individuales del pensamiento lógico y sus manifestaciones (Rubinstein, 1977; Leontiev, 1981; Labarrere, 1988, Pérez y Merino, 2008).

- Que el pensamiento tiene carácter problémico: para que surja la actividad del pensamiento es necesario que al individuo se le presente un problema, una 
interrogante que no pueda resolver por procedimientos conocidos. El pensamiento comienza por la necesidad de dar respuesta a una cuestión, de resolver un problema (Rubinstein, 1977; Labarrere, 1988).

- Que la actividad del pensamiento tiene carácter dirigido y consciente: no se piensa sobre algo en general, indefinido, sino sobre algo concreto, con un objetivo determinado. El pensamiento tiene carácter regulado (Rubinstein, 1977; Blanco, (2017).

- Que el pensamiento está relacionado con la actividad práctica, en ella surge y en ella se encuentra la comprobación de su veracidad. La actividad racional parte de la práctica y a ella vuelve para aplicar todo lo obtenido como resultado del pensamiento (Rubinstein, 1977; Flores, 2016).

- Que la solución de problemas ofrece potencialidades para el estudio de las características del pensamiento lógico del sujeto y se ofrecen las manifestaciones de cada una de las cualidades del pensamiento lógico en el proceso de solución de problemas de Física (Rodríguez, 2012).

- Que el estudio de las características del pensamiento lógico de los estudiantes permite estructurar el proceso de enseñanza-aprendizaje de manera que estos puedan alcanzar niveles superiores de desarrollo de forma progresiva (Travieso y Hernández, 2017).

En los estudios anteriores acerca del pensamiento lógico no se constatan antecedentes en los que se diseñen y operacionalicen dimensiones e indicadores de las cualidades de este proceso cognoscitivo a partir del proceso de solución de problemas, ni un proceder general para su caracterización con estas particularidades. Este elemento constituye la novedad de la investigación que desarrollan los autores del presente artículo.

En la solución de problemas interviene el pensamiento como proceso cognoscitivo en relación con otros procesos como las sensopercepciones, la memoria y la imaginación. Se coincide en señalar que el pensar es una actividad cognoscitiva de la personalidad; piensa el hombre con todos sus motivos, sentimientos y necesidades. No basta que ante el sujeto surja un problema para que él aplique la actividad del pensamiento, resulta imprescindible que en él surja la necesidad, el interés $y$ la posibilidad de resolverlo.

Generalmente, el trabajo del pensamiento en la solución de problemas requiere constancia y esfuerzo, lo que produce tensión y cansancio. Por lo anterior, supone la 
participación de la voluntad para continuar la búsqueda de los métodos según los cuales se pueda encontrar el resultado, con lo cual se produce más satisfacción cuando más esfuerzo requiere.

La mayoría de los investigadores coinciden en que todo proceso mental está orientado hacia la solución de problemas. El problema le proporciona el objetivo a la actividad pensante del hombre, lo que está vinculado con las condiciones de su planteamiento. Por tal motivo Rubinstein afirma que:

[...] El factor inicial del proceso mental es, por regla general, la situación problemática. El hombre empieza a pensar cuando siente la necesidad de comprender algo. El pensar empieza normalmente con un problema o con una cuestión, con un asombro o con una confusión, con una contradicción. (Rubinstein, 1966, p. 386)

La situación problémica es la que hace que se inicie el proceso mental, pero, en la formulación del problema a partir de esta, es necesario tener en cuenta las necesidades y motivos, los intereses de la persona estudiante; es decir, los aspectos emocionales que expresan las vivencias subjetivas del hombre, lo que se corresponde con el aspecto subjetivo del problema, entendido como interiorización de una contradicción para la que no se conocen las vías a emplear en su aclaración.

La peculiaridad del pensamiento, de expresarse predominantemente como solución de problemas, es reconocida por la mayoría de los autores. Según Labarrere (1996, p. 02) “La forma más peculiar y tal vez más importante de manifestarse el pensamiento es la solución y la formulación de problemas". El pensamiento en la solución de problemas se manifiesta, por tanto, como proceso de búsqueda, emisión de hipótesis, razonamientos, diseño de estrategias, comprobación de la solución y emisión de juicios.

Las operaciones del pensamiento que se manifiestan al resolver problemas están ampliamente tratadas en la bibliografía: Rubinstein (1977); Leontiev (1981); Labarrere (1988); Rodríguez, (2012); Flores (2016); Blanco, (2017); Pérez y Merino (2008), Travieso y Hernández (2017), destacándose que todo proceso mental es esencialmente analíticosintético, y este se manifiesta, de acuerdo con el nivel de complejidad del análisis y la síntesis en forma de abstracciones, generalizaciones y comparaciones. Cuando se quieren determinar las diferencias cualitativas en cuanto al desarrollo del pensamiento de una persona $\mathrm{u}$ otra se debe recurrir a las cualidades del pensamiento. En la literatura psicológica, de forma general, se relacionan las siguientes cualidades del pensamiento 
lógico: rapidez, profundidad, independencia, flexibilidad, consecutividad y amplitud. Sobre la base del análisis de la literatura antes referida, los autores de este artículo asumen la siguiente conceptualización de cada una de las cualidades del pensamiento lógico.

La rapidez del pensamiento es especialmente necesaria siempre que la persona se encuentre ante una situación en que la solución es inaplazable, es decir, aquella que tiene una limitación temporal prefijada. Es, además, en estos casos, cuando esta cualidad se pone de manifiesto, pues exige una solución acertada en un tiempo mínimo. Da la posibilidad de adoptar con prontitud una decisión adecuada. La rapidez está relacionada con los conocimientos, el grado de desarrollo de las habilidades intelectuales, del ritmo individual propio del modo de pensar. No debe confundirse con la precipitación en la solución de problemas que lleva a la ejecución sin un análisis profundo de la situación.

La profundidad del pensamiento permite penetrar en la esencia de los problemas, descubrir relaciones de causalidad en los fenómenos y procesos, no solo los más evidentes y cercanos, sino aquellas causas más lejanas y ocultas; es la capacidad de llegar a lo esencial y establecer nuevas generalizaciones, la habilidad de pronosticar la evolución posterior del hecho o fenómeno. La profundidad es considerada por algunos autores como la cualidad más importante del pensamiento.

La independencia del pensamiento permite al sujeto abordar el conocimiento de la realidad de manera creadora, original, buscar y encontrar nuevos medios de conocer la realidad, de formular y solucionar problemas, de plantear nuevas teorías y explicaciones. Esta cualidad expresa el carácter creador del pensamiento y está estrechamente vinculado con la crítica, o sea, la capacidad para no dejarse influir por otras vías de solución ya conocidas, de valorar los pensamientos ajenos y los propios con rigor y exactitud. El pensamiento crítico no acepta como cierta la primera solución, sino hasta comprobar su exactitud en la práctica. Esta cualidad es muy importante en el proceso de solución de problemas utilizando el método investigativo.

La flexibilidad del pensamiento da la posibilidad de cambiar los medios o vías de solución cuando estas resultan inadecuadas. Es saber encontrar nuevos caminos para estudiar un objeto, sin aferrarse a lo dado, sin atenerse al plan mental prefijado, cuando surgen condiciones que modifican las condiciones originales. Es decir, la flexibilidad se expresa en saber apreciar los cambios que exige un planteamiento nuevo del problema y de la solución de este. 
La consecutividad o logicidad del pensamiento permite al sujeto seguir un orden lógico de las acciones cuando se recapacita sobre un problema y se fundamentan y planifican mentalmente sus vías de solución. Es saber analizar la situación en forma sistémica, sin desviarse, sin saltar aleatoriamente de una idea a otra, observando un orden determinado. El pensamiento consecuente es eminentemente lógico, por eso algunos autores le llaman a esta cualidad logicidad.

La amplitud se manifiesta en la posibilidad de abarcar un mayor o menor círculo de cuestiones en la solución del problema, tanto de índole práctico como teórico, y de pensar de manera acertada y creadora sobre estos, de analizar multilateralmente la situación y los procedimientos de solución.

En la solución de los problemas se puede apreciar la manifestación de las cualidades antes relacionadas, donde están presentes más internamente las diferentes operaciones del pensamiento lógico. Debe destacarse que en el pensamiento se dan en interacción dialéctica las diferentes operaciones y cualidades de este proceso cognoscitivo y que la división que se ha hecho responde puramente a los fines didácticos propuestos en la investigación.

\section{Descripción de la metodología utilizada}

En esta investigación se siguió una metodología mixta en la que se tuvieron en cuenta métodos y técnicas tanto cuantitativas como cualitativas para el análisis y procesamiento de la información. Se elaboraron y aplicaron diferentes técnicas experimentales para evaluar el nivel de desarrollo del pensamiento lógico del estudiantado.

Para el estudio desarrollado, se define como variable cualitativa las cualidades del pensamiento lógico: rapidez, profundidad, independencia, flexibilidad, consecutividad y amplitud (cada una de estas cualidades constituye un indicador para el estudio). La variable cuantitativa está conformada por las puntuaciones asociadas a cada indicador, las escalas que se utilizan y los índices que se establecen. La determinación de estas variables permitió diseñar las técnicas experimentales, su tabulación y procesamiento.

Considerando que la función didáctica principal de los problemas es contribuir al desarrollo del pensamiento lógico del estudiantado, y que este proceso cognoscitivo se puede expresar a través de sus cualidades, los autores de este artículo proponen las siguientes dimensiones e indicadores para evaluar el nivel de desarrollo del pensamiento lógico. 


\section{I- $\quad$ Rapidez del pensamiento}

a) Rapidez en la formulación del problema:

(3) Alto. Si muestra rapidez de reacción ante la situación presentada, si arriba a la formulación adecuada del problema en el primer análisis, y si es capaz de fundamentar adecuadamente la estrategia utilizada.

(2) Medio. Si muestra rapidez de reacción ante la situación presentada, si arriba a la formulación adecuada del problema, aunque tenga que hacer algunas modificaciones a la primera formulación, y si es capaz de fundamentar adecuadamente la estrategia utilizada.

(1) Bajo. Si no puede arribar a la formulación del problema en el tiempo establecido, o prueba en más de dos oportunidades para arribar a la formulación adecuada sin llegar a fundamentar la estrategia seguida.

b) Rapidez en la solución del problema:

(3) Alto. Si muestra rapidez de reacción ante los problemas, si arriba a una respuesta correcta con la primera estrategia seleccionada, y si es capaz de fundamentar adecuadamente la estrategia utilizada.

(2) Medio. Si muestra rapidez de reacción ante los problemas, si arriba a una respuesta correcta aunque tenga que hacer algunas modificaciones a la estrategia seleccionada, y si la fundamenta adecuadamente.

(1) Bajo. Si no puede arribar a una respuesta correcta a los problemas en el tiempo establecido, o prueba más de dos estrategias para arribar a una respuesta correcta, sin llegar a fundamentarla estrategia seguida.

\section{II- Profundidad del pensamiento}

a) Determinar la esencia de la situación problémica:

(3) Alto. Si, a partir de tener en cuenta todos los aspectos esenciales del problema, llega a la solución acertada.

(2) Medio. Si omite algunos de los aspectos esenciales y llega solo parcialmente a la respuesta.

(1) Bajo. Si tiene en cuenta aspectos no esenciales y no llega a la solución correcta.

b) Considerar relaciones de causalidad en las condiciones del problema: 
(3) Alto. Si es capaz de determinar relaciones de causalidad en las condiciones del problema para llegar a la solución acertada.

(2) Medio. Si considera algunas relaciones de causalidad en las condiciones del problema y llega solo parcialmente a la respuesta.

(1)Bajo. Si no considera las relaciones de causalidad en las condiciones del problema y no llega a la solución correcta.

\section{III- Independencia del pensamiento}

a) Elaboración de nuevas estrategias de solución:

(3) Alto. Si elabora nuevas estrategias de solución de los problemas, aún cuando no se ajustan a los procedimientos explicados por la persona docente, para llegar a una solución correcta.

(2) Medio. Si resuelve el problema utilizando los mecanismos estudiados, sin necesidad de auxiliarse del profesor, los compañeros o la bibliografía.

(1) Bajo. Si para resolver el problema tiene que acudir reiteradamente al texto o pedir ayuda a otro compañero o al profesor.

b) Carácter crítico ante estrategias empleadas:

(3) Alto. Si analiza las estrategias de solución aplicadas individualmente o por sus compañeros críticamente, si destaca ventajas y desventajas, y si asume las más racionales o ingeniosas.

(2) Medio. Si analiza las estrategias de solución aplicadas individualmente o por sus compañeros críticamente, si destaca ventajas y desventajas, aunque no siempre asume las más originales o ingeniosas.

(1) Bajo. No analiza críticamente las estrategias de solución aplicadas.

\section{IV- Flexibilidad del pensamiento}

\section{a) Capacidad de rectificar las vías de solución inadecuadas:}

(3) Alto. Si es capaz de variar o modificar la estrategia de solución, en los casos que las condiciones del problema lo exigen, y llega a la respuesta correcta.

(2) Medio. Si es capaz de variar o modificar la estrategia de solución, en los casos que las condiciones del problema lo exigen, y llega parcialmente a la respuesta correcta. 
(1) Bajo. No es capaz de variar o modificar la estrategia de solución.

b) Posibilidad de empleo de varias vías de solución para un mismo problema:

(3) Alto. Si busca y encuentra más de una vía de solución a los problemas cuando las condiciones lo permiten.

(2) Medio. Si encuentra más de una vía de solución a los problemas cuando la persona docente se lo indica y le ofrece la ayuda necesaria.

(1) Bajo. No es capaz de encontrar más de una vía de solución a los problemas que logra resolver.

\section{V-Consecutividad del pensamiento}

a) Seguir un orden lógico en la solución del problema:

(3) Alto. Si se procede a la solución del problema siguiendo un orden lógico de los procedimientos para arribar a una respuesta correcta.

(2) Medio. Si procede a la solución del problema siguiendo un orden lógico cuando la estrategia es conocida.

(1) Bajo. Si procede a la solución del problema sin seguir un orden lógico.

b) Planificación de las acciones de solución:

(3) Alto. Si planifica adecuadamente las acciones de solución del problema de acuerdo con las condiciones para arribar a una respuesta correcta.

(2) Medio. Si planifica adecuadamente las acciones de solución del problema, pero no considera adecuadamente todas las condiciones.

(1) Bajo. No planifica adecuadamente las acciones de solución del problema, impone condiciones que hacen imposible su solución.

\section{VI- Amplitud del pensamiento}

a) Análisis integral del problema:

(3) Alto. Si analiza el problema en su integridad, y si considera todas las condiciones y posibles vías de solución para arribar a una respuesta correcta.

(2) Medio. Si analiza el problema en su integridad, si considera todas las condiciones y posibles vías de solución cuando el procedimiento es conocido para arribar a una respuesta correcta. 
(1) Bajo. Analiza elementos aislados del problema y descuida condiciones importantes que no le permiten encontrar vías de solución acertadas para llegar al resultado correcto.

\section{b) Capacidad de emplear los conocimientos, habilidades y hábitos asimilados en la solución de los problemas:}

(3) Alto. Si es capaz de hacer un análisis multilateral del contenido a emplear para la solución del problema y lo utiliza adecuadamente para arribar a una respuesta correcta.

(2)Medio. Si analiza elementos aislados del contenido a emplear para la solución del problema y llega parcialmente a la solución.

(1)Bajo. No emplea coherentemente el contenido asimilado en la solución de los problemas.

A partir de las dimensiones e indicadores definidos, es posible elaborar instrumentos (pruebas pedagógicas y técnicas experimentales de solución de problemas) para evaluar el desarrollo del pensamiento lógico de los estudiantes.

Para el estudio del desarrollo de las cualidades del pensamiento lógico se elaboraron y validaron, a través de un grupo de discusión, un conjunto de técnicas experimentales de solución de problemas. Debe tenerse en cuenta que la mayoría de las pruebas psicológicas para el estudio del pensamiento como la escala Binet-Simon, los test Terman-Merril, las escalas de inteligencia como WAIS y WISC se elaboran a partir de diferentes tipos de problemas. El objetivo de las técnicas fue estudiar el desarrollo de las cualidades del pensamiento a través de la actividad de solución de problemas.

Las técnicas experimentales de solución de problemas elaboradas consisten en una tarea o conjunto de tareas que se le plantean al estudiante con el fin de conocer sus individualidades en cuanto al desarrollo de las cualidades del pensamiento lógico. A cada una de estas técnicas se le asocia una norma de calificación con los índices correspondientes para lograr la mayor objetividad posible en la evaluación definitiva que se otorgue a cada estudiante. Algunas de estas se apoyan en la observación del proceso de solución de problemas por parte del investigador con el fin de complementar la información que brindan. 
La población está compuesta por los 37 estudiantes de la carrera de MatemáticaFísica de la Universidad de Ciego de Ávila. Se trabajó con una muestra no probabilística intencional de 12 estudiantes que ingresaron en el primer curso en 2016. Esta muestra se seleccionó atendiendo a la interacción constante de los autores con ellos. Se caracterizan por poseer motivación por la carrera escogida, dominio del contenido de la Física y de la Matemática de niveles precedentes, sin embargo presentaban limitaciones al enfrentar el proceso de solución de problemas.

Antes de aplicar las técnicas experimentales a la muestra de estudiantes seleccionada se les explicaron las características de la investigación que se estaba desarrollando, sus objetivos y los beneficios que podía aportar para perfeccionar sus aprendizajes. Asimismo, se les solicitó su colaboración voluntaria para participar, se logró el consentimiento de los 12 estudiantes previstos. Inmediatamente concluido este proceso, se procede a la aplicación de las técnicas experimentales diseñadas.

En la primera técnica, se les ofrecen seis letras para que durante dos minutos escriban el mayor número de palabras posibles que se usan en Física General, con el objetivo de valorar la amplitud del pensamiento; en la segunda técnica, se les dan cuatro palabras de las que se usan en la misma disciplina y se les orienta a escribir todas las oraciones posibles empleando el tiempo que necesiten para poder valorar cualidades como la amplitud, la consecutividad y la rapidez; por último, en la tercera técnica se dan diferentes situaciones, preguntas que deben responder de forma lógica y algunos problemas para evaluar el resto de las cualidades.

Para evaluar con la mayor objetividad posible cada una de las cualidades, se establecieron las siguientes puntuaciones para calificar cada indicador: 3 (alto), 2 (medio) y 1 (bajo) y para la calificación de estas técnicas se estableció una norma que las integrara a todas.

Con la norma establecida se elaboró y validó con un coeficiente Alpha de Cronbach de 0,7 la escala establecida (Ver anexo 1), lo que permitió hacer una valoración cualitativa global de las características del desarrollo de las cualidades del pensamiento lógico de los 12 estudiantes muestreados. Debe destacarse que en las técnicas experimentales, basadas en la solución de problemas, se siguieron los pasos que a continuación se mencionan para, a través de la observación y el análisis de los protocolos, llegar a conclusiones y obtener una información más directa acerca de las características de las cualidades del pensamiento lógico estudiadas: 
- Se les propone el problema para que lo resuelvan individualmente.

- Se recogen los trabajos de aquellos estudiantes que terminan de forma correcta según el tiempo de entrega.

- Se forman equipos con los que no arriban a soluciones correctas y se orienta continuar la búsqueda, ahora en colectivo.

- Se recogen los resultados de los equipos indicando el tiempo.

- Se les pide a algunos estudiantes que escriban el procedimiento realizado o se establece un debate colectivo sobre el trabajo realizado por los diferentes equipos y por estudiantes individualmente.

Durante la aplicación de las técnicas experimentales (al final del artículo se recoge una muestra de ellas) los investigadores, por medio de la observación, lograron tomar la mayor cantidad de información acerca de la actividad y el comportamiento de los estudiantes en el proceso de solución de los problemas, se anotaron las preguntas que hacían, el tiempo en que entregaban los protocolos, el nivel de ayuda necesario en cada caso, para, posteriormente con esta información y los trabajos de los alumnos, proceder a dar una calificación en correspondencia con los índices adoptados para cada cualidad del pensamiento lógico, de forma que se pudieran reducir al mínimo posible los factores subjetivos.

Para otorgarle el valor cuantitativo final a cada cualidad del pensamiento, a partir de sus respectivos indicadores, se procedió con el siguiente criterio establecido convencionalmente por los autores:

- Tendrá alto (3) cuando se den las combinaciones: alto (3) y alto (3) o alto (3) y medio (2).

- Tendrá medio (2) cuando se den las combinaciones: medio (2) y medio (2) o medio (2) y bajo (1).

- Tendrá bajo (1) cuando obtenga bajo en dos de los indicadores.

\section{Análisis de los resultados}

En la Tabla 1 aparecen los resultados de las técnicas aplicadas, en ella se refleja la cantidad de alumnos por puntuaciones en cada indicador. 
Tabla 1

Resultados de la aplicación de las técnicas experimentales a los 12 estudiantes de la carrera de Matemática-Física de la Universidad de Ciego de Ávila, Cuba, por puntuaciones de cada indicador. Octubre de 2016.

\begin{tabular}{|r|r|r|r|r|}
\cline { 2 - 5 } \multicolumn{1}{c|}{ Cantidad De Estudiantes Por Índice } \\
\hline Indicadores & Bajo & Medio & Alto & Total \\
\hline Rapidez & 6 & 4 & 2 & 12 \\
\hline Profundidad & 6 & 5 & 1 & 12 \\
\hline Independencia & 5 & 4 & 3 & 12 \\
\hline Flexibilidad & 5 & 3 & 4 & 12 \\
\hline Consecutividad & 4 & 2 & 6 & 12 \\
\hline Amplitud & 5 & 4 & 3 & 12 \\
\hline
\end{tabular}

Fuente: Elaboración propia con información obtenida de los estudiantes participantes, 2016.

El análisis de la Tabla 1 revela que las cualidades del pensamiento lógico con mayores limitaciones en la muestra estudiada son la profundidad y la rapidez. Este hecho se expresa en que los estudiantes arriban a soluciones correctas de los problemas planteados utilizando un tiempo superior al fijado y en que, en el análisis de las condiciones de estos, se dejan de establecer algunas relaciones causales esenciales que provocan que no se llegue a la solución correcta.

Con el objetivo de lograr una interpretación más clara de los resultados cuantitativos obtenidos se determinó el índice promedio para cada indicador $\left(\mathrm{I}_{\mathrm{c}}\right)$, el cual expresa el valor que alcanza en una escala ordinal de 1 a 3 en un momento determinado.

$I_{c}=\left(3 I_{1}+2 I_{2}+I_{3}\right) / n$

Donde $I_{c} e s$ el índice promedio para el indicador de la muestra.

$I_{1}, I_{2}, I_{3}$ designa la cantidad de estudiantes con 3,2 y 1 respectivamente en cada indicador.

$\mathrm{n}$ es el total de estudiantes muestreados.

Posteriormente, se determinó el índice porcentual ( 1 c\%) que indica en qué por ciento de la escala adoptada se encuentra el índice de la muestra en el momento de la aplicación de la evaluación.

$I_{c \%}=$ (Ic / 3 n) $100 \%$

Donde lc es el índice promedio para el indicador de la muestra. 
En la Tabla 2 aparecen los resultados resumidos de los valores del índice de la muestra y el índice porcentual.

Tabla 2

Tabulación de los índices para la muestra de 12 estudiantes de la carrera de Matemática-Física de la Universidad de Ciego de Ávila, Cuba, por puntuaciones de cada indicador. Octubre de 2016.

\begin{tabular}{|r|r|r|}
\hline Indicador & $\mathrm{I}_{\mathrm{c}}$ & $\mathrm{I}_{\mathrm{c}(\%)}$ \\
\hline Rapidez & 1,66 & 4,61 \\
\hline Profundidad & 1,58 & 4,38 \\
\hline Independencia & 1,83 & 5,08 \\
\hline Flexibilidad & 1,91 & 5,30 \\
\hline Consecutividad & 2,16 & 6,00 \\
\hline Amplitud & 1,83 & 5,08 \\
\hline
\end{tabular}

Fuente: Elaboración propia con información obtenida de los estudiantes participantes, 2016.

La evaluación de cada indicador en la etapa correspondiente según el criterio establecido se realizó teniendo en cuenta la siguiente escala a partir del análisis del índice promedio para cada indicador:

- $\quad$ de 1 a 1,59 - bajo (B)

- de 1,6 a 2,09 - medio (M)

- $\quad$ de 2,1 a 3 -medio alto (A)

Como se puede apreciar el indicador de consecutividad del pensamiento se evalúa de alto y el resto de los indicadores se encuentran en el nivel medio de desarrollo, excepto el indicador profundidad que está en el nivel bajo. Los estudiantes de la muestra demuestran logicidad en la solución de los problemas planteados al seguir un orden adecuado de las acciones de formulación, análisis de la solución, solución y comprobación de los resultados. Sin embargo, manifiestan limitaciones en el resto de las cualidades examinadas. El índice porcentual $(\mathrm{I} \%)$ indica en qué por ciento de la escala adoptada se encuentra el índice de la muestra en el momento de la aplicación de la evaluación, se aprecia que los indicadores que se encuentran a un porciento menor son la rapidez y la profundidad del pensamiento, por tanto son los que presentan mayores limitaciones.

Las regularidades principales constatadas con la aplicación de las técnicas elaboradas son las siguientes: 
- Los estudiantes de la muestra siguen un orden lógico en las acciones de formulación, análisis de la solución, solución y comprobación de los resultados de los problemas planteados.

- Los estudiantes manifiestan dificultades en determinar algunas relaciones de causalidad en el enunciado de los problemas, en llegar a la esencia de la situación problémica y en hacer generalizaciones de carácter teórico, lo que demuestra insuficiencias en la profundidad del pensamiento.

- Para algunos estudiantes es difícil encontrar, con independencia, nuevos criterios para resolver el problema, plantear nuevas explicaciones, hacer el análisis crítico de las situaciones presentadas. Finalmente, se dejan influir con gran facilidad por el sentido común.

- Se manifiestan dificultades en el proceso de solución cuando es necesario hacer cambios en las condiciones que modifican los algoritmos o procedimientos prefijados, lo que lleva al abandono de la búsqueda de la solución sin encontrar nuevos caminos, consecuentemente, se aferran a lo ya conocido.

\section{Conclusiones}

En los estudios anteriores acerca de la exploración del desarrollo del pensamiento lógico de los estudiantes no se constatan antecedentes en los que se definan y operacionalicen dimensiones e indicadores de las distintas cualidades de este proceso cognoscitivo a partir del proceso de solución de problemas que permitan diseñar técnicas experimentales para su caracterización efectiva. Este elemento constituye la novedad de la investigación que desarrollan los autores del presente artículo.

En esta investigación se determinan las dimensiones y los indicadores que permiten caracterizar el desarrollo de las cualidades del pensamiento lógico en una muestra de 12 estudiantes de la carrera de Matemática-Física de la Universidad de Ciego de Ávila, Cuba, tomando como referencia el proceso de solución de problemas de Física. Este elemento posibilitó diseñar y validar un proceder para caracterizar el desarrollo del pensamiento lógico del estudiantado.

La metodología empleada (enfoque mixto) permitió establecer como variable cualitativa las cualidades del pensamiento lógico: rapidez, profundidad, independencia, flexibilidad, consecutividad y amplitud (cada una de estas cualidades constituye un indicador 
para el estudio), mientras que la cuantitativa está conformada por las puntuaciones asociadas a cada indicador, las escalas que se utilizan y los índices que se establecen.

Una caracterización previa de la muestra reveló que los estudiantes poseen motivación por la carrera escogida y dominio del contenido de la Física y de la Matemática de niveles precedentes. No obstante, presentaban limitaciones al enfrentar el proceso de solución de problemas.

Un análisis factico de los resultados obtenidos con la aplicación de las técnicas experimentales utilizadas evidencia limitaciones en las cualidades del pensamiento de rapidez y profundidad, pues se constata que los estudiantes de la muestra pueden arribar a soluciones correctas de los problemas planteados utilizando un tiempo superior al fijado (6 estudiantes) y que en el análisis de las condiciones de los problemas se dejan de establecer algunas relaciones causales esenciales que provocan que no se llegue a la solución correcta (6 estudiantes).

Los resultados del análisis de los índices cuantitativos establecidos para la muestra evidenciaron que la cualidad de consecutividad del pensamiento se encuentra en un nivel alto de desarrollo, mientras que las demás cualidades en un nivel medio, de acuerdo con los valores de dichos índices que fueron de 2,16 para la consecutividad, y se comportaron entre 1,6 y 2 para la mayoría de las cualidades, con excepción de la profundidad del pensamiento que alcanzó solo el valor de 1,58.

El análisis cualitativo de los resultados demostró que los estudiantes de la muestra siguen un orden lógico en las acciones de formulación, análisis de la solución, solución y comprobación de los resultados de los problemas planteados. A pesar de ello, se manifiestan dificultades en el proceso de solución cuando se necesita realizar transformaciones en las condiciones que modifican los procedimientos prefijados.

La investigación realizada demostró que existe un adecuado nivel de desarrollo del pensamiento lógico en los y las estudiantes que ingresan a la universidad en la carrera de Matemática-Física, expresado fundamentalmente en la cualidad de consecutividad, el resto de las cualidades del pensamiento lógico está a un nivel medio de desarrollo (rapidez, independencia, flexibilidad y amplitud) exceptuando la profundidad del pensamiento que se comportó en un nivel bajo de desarrollo. Los resultados obtenidos permitieron diseñar las estrategias de aprendizaje de acuerdo con las peculiaridades del desarrollo intelectual de los estudiantes. 


\section{Referencias}

Blanco, Rafael. (2017). El pensamiento lógico desde las perspectivas de las neurociencias cognitivas (Tesis para optar por el grado de Doctor en Filosofía). Universidad de Oviedo. Recuperado de www.eikasia.es/documentos/rafaelblanco.pdf

Flores, Yesika. (11 de enero, 2016). Pensamiento lógico [Mensaje en un blog]. Recuperado de http://style.shockvisual.net/pensamiento-logico/

Labarrere, Alberto. (1988). Bases psicológicas de la enseñanza de la solución de problemas matemáticos en la escuela primaria. La Habana: Editorial Pueblo y Educación.

Labarrere, Alberto. (1996). Pensamiento, análisis y autorregulación de la actividad cognoscitiva de los alumnos. La Habana: Editorial Pueblo y Educación.

Leontiev, Alekséi. (1981). Actividad, conciencia, personalidad. La Habana: Editorial Pueblo y Educación.

Pérez, Julián y Merino, María. (12 de junio, 2008). Definición de pensamiento lógico [Mensaje en un blog]. Recuperado de https://definicion.de/pensamiento-logico/

Rodríguez, Luis. (2012). Metodología para la solución de problemas en el proceso de enseñanza-aprendizaje de las ciencias en la escuela. En Temas seleccionados de Didáctica de la Física (pp. 170-179). La Habana: Editorial Pueblo y Educación.

Rubinstein, Serguéi. (1977). Principios de Psicología General. La Habana: Editorial Pueblo y Educación.

Rubinstein, Serguéi. (1966). El proceso del pensamiento. La Habana: Editorial Universitaria.

Travieso, Dayana y Hernández, Adela. (2017). El desarrollo del pensamiento lógico a través del proceso de enseñanza aprendizaje. Revista Cubana de Educación Superior, 36 (1), 53-58. Recuperado de http://scielo.sld.cu/scielo.php?script=sci arttext\&pid=S0257$\underline{43142017000100006}$ 


\section{Anexo 1}

Ejemplo de técnicas experimentales utilizadas con las correspondientes normas de calificación.

\section{Instrumento 1:}

1-. Se le ofrecen varias letras. Debe escribir, durante dos minutos, el mayor número de palabras posibles que se usen en las clases de Física y que comiencen con estas letras.

\section{Letra A:}

Letra F:

Letra $\mathrm{M}$ :

Letra S:

Letra L:

Letra R:

\section{Instrumento 2:}

Le relacionamos varias palabras empleadas en las clases de Física. Elabore todas las oraciones que pueda con cada una de ellas. Disponga del tiempo necesario.

MASA:

ENERGÍA:

VELOCIDAD:

MOVIMIENTO:

\section{Instrumento 3:}

Técnicas experimentales empleadas para valorar las habilidades y cualidades del pensamiento lógico relacionadas con las fases de ejecución y control valorativo. 
a) ¿Cómo determinar la velocidad inicial de una bala disparada por una pistola de juguete si cuenta solo con una cinta métrica?

Este problema se puede resolver utilizando diferentes variantes, es decir, por el método cinético o energético y dentro de ellos se pueden realizar diseños variados.

b) ¿Cómo determinar la velocidad inicial de una bala disparada por una pistola de juguete si cuenta solo con un cronómetro?

Igual que el problema anterior, se puede resolver por diferentes variantes.

c) ¿Cómo determinar el coeficiente de fricción entre una chapa metálica y una varilla de madera utilizando solamente una regla graduada?

Una variante para resolverlo puede ser apoyando en la chapa metálica la varilla de madera verticalmente y comenzar a inclinarla paulatinamente, presionando su extremo superior. A un ángulo de inclinación determinado, la varilla comienza a deslizarse sobre el metal. En este momento la componente horizontal de la fuerza es igual a la fuerza máxima de rozamiento estático, de donde se puede obtener una expresión para determinar este último en función de los catetos del ángulo formado por la varilla con la horizontal en el momento en que se comienza a deslizar.

d) ¿Cómo determinar la carga que puede soportar un hilo con la ayuda de un cuerpo de masa conocido, un soporte cualquiera y un compás?

Para resolver este problema debe colgarse el cuerpo de masa conocida del centro del cordel y sostener sus extremos a un soporte. Tirando paulatinamente del hilo se aumenta el ángulo y como resultado crece la fuerza de tensión. Al marcar con el compás el ángulo en que se rompe el cordel se puede calcular la carga que soporta, aplicando las leyes de Newton. e) ¿Cómo determinar el ángulo de inclinación de un plano si cuenta con un bloque de madera y un dinamómetro? Si el bloque de madera está en reposo con respecto al plano. Una variante para resolverlo puede ser determinando con el dinamómetro la fuerza necesaria para que el bloque se mueva con velocidad aproximadamente constante hacia arriba y hacia abajo, de donde se puede obtener, aplicando las leyes de Newton, una expresión para calcular el seno del ángulo en función de estas fuerzas y la masa del cuerpo utilizado.

f) ¿Cómo determinar la masa de un cuerpo si cuenta con un resorte y otro cuerpo de masa conocida? 
Estos problemas permitieron hacer valoraciones cualitativas acerca de las siguientes habilidades que son un reflejo del desarrollo de las cualidades del pensamiento lógico de los estudiantes:

- Hacer un análisis profundo del problema.

- Operar con las componentes del problema y sus representaciones.

- Operar con símbolos y fórmulas.

- Buscar diferentes vías de solución.

- Encontrar la vía más racional.

- Realizar el análisis y ejecutar las operaciones con independencia.

- Mostrar un carácter reflexivo y consecuente de las acciones.

- Planificar las operaciones.

- Idear métodos de solución no estudiados.

- Ver diferentes alternativas en los procedimientos de solución de un mismo problema.

- Control de las acciones y el resultado.

- Valorar aspectos positivos y negativos en el proceso de solución y ejecución de los problemas.

II- Norma de calificación utilizada para evaluar las cualidades del pensamiento lógico de los alumnos en las técnicas que se basan en la solución de problemas.

Problema:

¿Cómo determinar la velocidad inicial de una bala disparada por una pistola de juguete si cuenta solo con una cinta métrica?

PROFUNDIDAD:

(3) - Si a partir de tener en cuenta todos los aspectos esenciales llega a la solución correcta.

- Muestra claridad de objetivos a partir de una buena comprensión.

- Pronostica en el rango que debe estar la solución.

- Realiza un análisis profundo del problema.

- Determina relaciones de causalidad en las condiciones del problema.

(2) - Omite algunos de los aspectos esenciales y llega solo parcialmente a la respuesta (llega a las ecuaciones a emplear para una vía determinada, pero no logra diseñar correctamente el experimento a realizar).

- Muestra claridad de objetivos pero no llega totalmente al resultado esperado. 
- Realiza un análisis adecuado de las condiciones del problema pero omite algunos elementos en el diseño de la estrategia.

- No considera adecuadamente todas las relaciones de causalidad en las condiciones.

(1) - Centra el análisis en aspectos no esenciales y no llega a la solución correcta.

- Muestra tendencia a la ejecución y no acierta en el método escogido.

- No considera las relaciones de causalidad fundamentales

INDEPENDENCIA:

(3) - Elabora una estrategia correcta y llega a la solución adecuada de forma independiente.

- En el debate es capaz de encontrar otras estrategias de solución y asume la más racional.

(2) - Resuelve el problema con una estrategia adecuada pero necesita ayuda del profesor o los compañeros.

- En el debate puede fundamentar elementos aislados del procedimiento que pensó.

- Analiza las estrategias críticamente aunque no siempre asume las más ventajosas.

(1) - Para resolver el problema necesita una atención directa del profesor o los compañeros y no logra fundamentar ningún elemento del procedimiento seguido.

- No analiza críticamente las estrategias empleadas.

FLEXIBILIDAD:

(3) - Cuando se percata de que la estrategia adoptada no es correcta la varía de modo independiente.

- Encuentra al menos dos vías de solución.

- En el debate valora aspectos positivos y negativos de las soluciones y asume las más racionales.

(2) - Solo puede encontrar una vía de solución distinta a la pensada bajo la dirección del profesor o los compañeros.

- En el debate valora aspectos aislados en el enjuiciamiento de los diferentes procedimientos empleados.

(1) - No es capaz de variar o modificar la estrategia de solución por lo que no puede arribar a una respuesta correcta, se aferra a la primera vía pensada aunque no sea adecuada. 


\section{CONSECUTIVIDAD:}

(3)- Si sigue un orden lógico en la solución del problema y llega a la solución esperada.

- Realiza adecuadamente las transformaciones necesarias.

- Opera correctamente con las fórmulas.

- Planifica adecuadamente las acciones.

(2) - Si sigue un orden lógico de solución cuando la estrategia es conocida.

- Realiza adecuadamente las transformaciones y operaciones pero no considera adecuadamente todas las condiciones.

(1) - Procede en la solución del problema sin seguir un orden lógico y sin llegar a planificar adecuadamente las acciones imponiendo condiciones que hacen imposible su solución.

\section{AMPLITUD:}

(3) - Analiza el problema en su integralidad, considera todas las condiciones y posibles vías de solución para arribar a una respuesta correcta.

- En el debate asimila otras vías de solución y asume las más racionales.

- Aplica adecuadamente el contenido asimilado a la situación concreta del problema.

(2) - Analiza el problema en su integralidad, considera todas las condiciones y posibles vías de solución cuando el procedimiento es conocido.

- Aplica elementos aislados del contenido asimilado a la situación concreta del problema. (1) - Analiza elementos aislados del problema, descuida condiciones importantes que no le permiten encontrar las vías acertadas, diseñar la estrategia, planificar y ejecutar las acciones correspondientes.

- No emplea coherentemente el contenido asimilado a la solución del problema. 